Larruzea-Urkixo, N. y Cardeñoso Ramírez, O. (2020). Diferencias individuales en aprendizaje autorregulado de estudiantes de los Grados de Educación: género, especialidad, notas y desempeño académico. Revista de Investigación Educativa, 38(2), 453-473.

DOI: http://dx.doi.org/10.6018/rie.334301

\title{
Diferencias individuales en aprendizaje autorregulado de estudiantes de los Grados de Educación: género, especialidad, notas y desempeño académico
}

\author{
Individual differences in self-regulated learning among Education \\ Degree students: gender, teaching specialty, grades and academic \\ performance
}

\author{
Nerea Larruzea-Urkixo y Olga Cardeñoso Ramírez \\ Universidad del País Vasco (UPV-EHU)
}

\begin{abstract}
Resumen
Introducción: conocer las diferencias en los procesos de autorregulación del aprendizaje del alumnado actual es clave para la mejora de la formación en nuestras universidades. El objetivo de este estudio es analizar la variabilidad en dichos procesos en función del género, la especialidad, las notas (de acceso y de grado) y otras variables de desempeño académico. Método: participaron en el estudio 456 estudiantes (335 mujeres y 119 hombres) de los Grados en Educación Infantil y Primaria que completaron la versión en español del Motivated Strategies for Learning Questionnaire (Ramírez, Canto, Bueno \& Echezarreta, 2013) junto a datos relativos al desempeño académico. Resultados: Se mostró la existencia de diferencias en aprendizaje autorregulado en función del género, pero no de la especialidad ni de la interacción entre género*especialidad. También se reveló que las alumnas poseían mayor autorregulación que los alumnos tanto en variables motivacionales como en estrategias de aprendizaje. A continuación, se hallaron diferencias en aprendizaje autorregulado en función de la nota de acceso, de grado y de la interacción género*nota de grado. Aunque de manera general estos datos confirman que "a mayor nota de

Correspondencia: Olga Cardeñoso Ramírez, Departamento de Psicología Evolutiva y de la Educación, Facultad de Educación de Bilbao, Universidad del País Vasco, Barrio Sarriena s/n, 48940, Leioa, Bizkaia, Spain. Tel: +34 (946014601) Correo electrónico: mariaolga.cardenoso@ehu.eus.
\end{abstract}


acceso, mejor autorregulación", los resultados desvelaron un declive del alumnado con mejores notas de grado en varias subescalas exceptuando en aprendizaje entre iguales. Finalmente, se mostró que las alumnas perciben la carrera con mayor dificultad, dedican más horas y presentan un mejor rendimiento académico de lo esperado. Discusión: Estas diferencias entre el alumnado deberían ser consideradas para potenciar la autorregulación en las aulas.

Palabras clave: estrategias de aprendizaje; motivación; diferencias de género; rendimiento académico; formación de docentes.

\section{Abstract}

Introduction: Identifying differences in self-regulatory processes among current students is key to improve training in our universities. The aim of this study is to analyze the variability in the aforementioned processes according to gender, teaching specialty, grades (admission grade and grade point average, GPA) and other variables related to academic performance. Method: 456 Primary Education and Early Childhood Education students participated in the study (335 women and 119 men) by completing the Spanish version of the Motivated Strategies for Learning Questionnaire (Ramírez, Canto, Bueno \& Echezarreta, 2013). Results: Differences were found in self-regulated learning based on gender, but not on specialty or on the interaction between gender*specialty. It was also shown that female students had greater self-regulatory skills than male students in both motivational variables and learning strategies. Besides, differences were found in self-regulated learning according to admission grade, grade point average and the interaction gender*grade point average. Although, overall, the data obtained confirm that "the higher the admission grade, the better self-regulatory skills", results revealed a decline among students with better grades in several subscales except for peer learning. Finally, it was shown that female students have a higher awareness of the degree's difficulty, dedicate more hours and present a better academic performance than expected. Discussion: These differences between students should be considered in order to promote self-regulation in the classroom.

Keywords: learning strategies; motivation; gender differences; academic performance; teacher education.

\section{Introducción}

La sociedad actual se encuentra inmersa en un período de rápidas transformaciones culturales, económicas, tecnológicas y sociales (Vargas, 2017). Estos cambios globales se manifiestan también en el sistema educativo, entre otros aspectos, a través de la incorporación del enfoque basado en competencias. Este nuevo modelo incluye no sólo la enseñanza de carácter científico, sino también la adquisición de competencias transversales encaminadas fundamentalmente a que el alumnado aprenda a desenvolverse de manera autoconsciente y autodirigida en el modelo de sociedad actual (Camoiras, Benito \& Varela, 2018).

De acuerdo con Zimmerman (2013) este tipo de aprendizaje, denominado autorregulado, se define como un proceso a través del cual las personas activan y mantienen cogniciones, conductas y afectos dirigidos sistemáticamente hacia el logro de sus metas, teniendo en cuenta sus posibilidades y limitaciones. La autorregulación en el aprendizaje incluye elementos tanto de motivación como de estrategias de aprendizaje 
y hace hincapié en la importancia de contextualizar los procesos de aprendizaje en los ámbitos específicos de socialización del alumnado (Ben-Eliyahu \& Bernacki, 2015; BenEliyahu \& Linnenbrink-Garcia, 2015; Karabenick \& Zusho, 2015). Esto nos sugiere la conveniencia de analizar las diferencias individuales explorando elementos relativos al contexto, como en este estudio, los grados de educación.

Existen otras variables que también están relacionadas con las diferencias en las habilidades de autorregulación en el aprendizaje, fundamentalmente, el género y el rendimiento académico.

Con respecto al género, la investigación indica que existen diferencias entre chicos y chicas (Bozpolat, 2016; Muñoz, 2012; Torrano, Fuentes \& Soria, 2017; Tseng, Liu \& Nix, 2017; Valenzuela \& Suárez, 2017) y que estas aparecen incluso desde la primera infancia (Ponitz, McClelland, Mattheews \& Morrison, 2009). Ya en 1990, autores referentes en el origen y posterior desarrollo de la teoría del aprendizaje autorregulado como Zimmerman y Martínez-Pons hallaron que las chicas hacían un mayor uso de estrategias de aprendizaje relacionadas con la planificación, el establecimiento de metas, el control y la optimización del contexto. En la misma línea, Ablard y Lipschultz (1998) confirmaron que las estudiantes presentaban un mayor uso en las estrategias implicadas en la regulación personal y optimización del contexto. Bidjerano (2005), por su parte, también mostró que las estudiantes empleaban en mayor medida estrategias de repetición, organización, autorregulación metacognitiva, control del tiempo, elaboración y regulación del esfuerzo de cara a conseguir sus metas académicas, en línea con lo hallado por Navea (2015) y con Torrano y Soria (2017). En lo que respecta específicamente a la autorregulación metacognitiva, las alumnas también gestionan y controlan mejor su propio pensamiento (Albert, 2017; Suárez, Anaya \& Gómez, 2004).

Estas diferencias de género también están relacionadas con la motivación. Las alumnas poseen menores niveles de motivación extrínseca que sus compañeros (Rusillo \& Casanova, 2004), mayor motivación intrínseca y ansiedad ante los exámenes (Albert, 2017), así como creencias de autoeficacia (Rianudo, Barrera \& Donolo, 2006) y creencias de control (Navea, 2015).

En relación a la segunda variable, el rendimiento académico, la investigación ha revelado en múltiples ocasiones que el alumnado universitario con mayor rendimiento académico presenta una mejor regulación en las distintas dimensiones y procesos implicados en el aprendizaje autorregulado frente al alumnado con peor rendimiento o dificultades en el aprendizaje (Broadbent \& Poon, 2015; Dörrenbächer \& Perels, 2016; García-Ros \& Pérez-González, 2011).

Aunque escasas, algunas investigaciones han sido planificadas teniendo en cuenta al mismo tiempo tanto las mencionadas diferencias asociadas al género como las que tienen relación con el rendimiento académico. Salmerón, Gutiérrez-Braojos y Rodríguez (2017), por ejemplo, mostraron puntuaciones significativamente superiores por parte de las estudiantes en regulación interna, así como en la nota media de grado.

En lo referente a la nota de acceso, García-Ros y Pérez-González (2011) también hallaron que las alumnas poseían mejores notas. En esta línea, Dörrenbächer y Perels (2016) hicieron una distinción entre la nota de acceso y de grado, revelando que el alumnado con mayor motivación y más habilidades obtenía mejores notas. Tal y 
como reflexiona Bussey (2015), estas diferencias de género en rendimiento académico pueden deberse a factores motivacionales resultantes de influencias sociales que promueven estereotipos de género tradicionales basados en la responsabilidad $\mathrm{y}$ el sacrificio.

Los componentes del aprendizaje autorregulado, fundamentales en la autorregulación en el aprendizaje de todo el alumnado universitario, cobran aún mayor importancia cuando se trata del futuro profesorado. Tal y como apuntan Chocarro, González-Torres y Sobrino (2007), la sociedad actual demanda profesionales de la educación cada vez más cualificados y competentes, capaces de actualizar sus destrezas y conocimientos contínuamente. Por ello, el estudio del aprendizaje autorregulado en los Grados de Educación es un factor clave dada la capacidad de enseñanza y promoción de las habilidades de autorregulación del profesorado en su alumnado (Peeters et al., 2014; Randi, Corno \& Johnson, 2011), tal y como se ha mostrado en varias investigaciones (Perels, Dignath \& Schmitz, 2009; Perry \& Vandekamp, 2000). Todo esto pone de relieve la importancia de avanzar en el estudio de las habilidades autorregulatorias en la formación docente (Chocarro et al., 2007), tal y como se plantea en este trabajo.

A modo de resumen podemos constatar que hay estudios que indagan en el papel del género en la autorregulación, otros en la relación entre las notas (acceso o grado) y la autorregulación, y sólo algunos incluyen el análisis de ambos factores y su interacción. Asimismo, no hay estudios que analicen si la especialidad del grado de educación cursada cursada se relaciona con diferencias en autorregulación, y pocos trabajos incluyen como variables otros componentes de desempeño general que pueden afectar a las habilidades autorregulatorias en la universidad. Entre ellos, la compaginación o no de los estudios con trabajos remunerados (García-Ros \& PérezGonzález, 2011), el número de horas de dedicación fuera del aula o la dificultad percibida de la carrera como la expectativa ante la misma. Por este motivo, en este estudio, se incluyen el género, las notas (acceso y grado), la especialidad y variables de desempeño académico.

\section{Método}

\section{Objetivos}

Tomando en consideración la literatura previa, esta investigación propone los siguientes objetivos: 1. Analizar posibles diferencias en función del género, de la especialidad cursada y de la interacción género*especialidad en los diferentes procesos de aprendizaje autorregulado del alumnado de los grados de Educación; 2. Analizar diferencias en aprendizaje autorregulado en función del rendimiento académico (notas de acceso y de grado), del género y de la interacción entre rendimiento* género y 3. Explorar la relación del género con variables de desempeño académico como el tiempo de estudio, la dificultad percibida, las horas de dedicación, el trabajo y la asistencia a clase, ampliando el análisis de las conductas observadas del alumnado. 
En base a los objetivos, este estudio plantea las siguientes hipótesis: 1 . Se espera encontrar diferencias a favor de las mujeres en la mayoría de componentes del aprendizaje autorregulado; y 2. Se espera obtener que el alumnado con mejores habilidades de autorregulación presente un mayor rendimiento académico. No es posible plantear hipótesis con respecto a las diferencias de género en las notas de acceso, grado ni en variables de desempeño académico dado que no se han hallado estudios previos en las que sean abordadas.

\section{Población y Muestra}

La muestra empleada para esta investigación consta de 456 estudiantes de primer y segundo curso del Grado de Educación Primaria y del Grado de Educación Infantil de la Facultad de Educación de Bilbao de la Universidad del País Vasco (UPV/EHU) en España, seleccionados de manera incidental. Concretamente, 119 hombres $(26.1 \%)$ y 335 mujeres (73.5\%) y dos personas que no especificaron su género (0.4\%). 293 de estos participantes pertenecían al Grado de Educación Primaria: 187 alumnas (41\% del total) y 105 alumnos (23.06\% del total) y, 163 al Grado de Educación Infantil, en concreto, 148 mujeres (32.45\%) y 14 hombres (3.07\%). La muestra estuvo formada por el alumnado que asistió a clase el día de la cumplimentación de las pruebas.

\section{Instrumento}

El Motivated Strategies of Learning Questionnaire (MSLQ, Pintrich, Smith, Duncan \& Mckeachie, 1991), adaptado por Ramírez, Canto, Bueno y Echezarreta (2013) se trata de un instrumento de autoinforme diseñado para evaluar las orientaciones motivacionales y el uso de diferentes estrategias de aprendizaje del alumnado universitario en una materia/curso. Se ha optado por esta adaptación debido a que se prescinde de un menor número de ítems y la estructura factorial subyacente concuerda en mayor medida con la propuesta original y el modelo conceptual de aprendizaje autorregulado de Pintrich et al. (1991) al compararla con otras adaptaciones al español como el CEAM-II (Roces, Tourón y González-Torres, 1995).

Además, el proceso de la adaptación utilizada siguió las directrices establecidas por la International Test Commission (ITC). Si bien es una versión diseñada en el contexto mexicano, se ha mostrado el buen funcionamiento de instrumentos latinoamericanos con población española, y viceversa, en otros estudios (Cardozo, 2008). Concretamente, en el trabajo de López de la Serna (2016), con una muestra similar a la de este estudio, se hizo uso de la versión previa del instrumento de medida empleado en esta investigación, por lo que se puede concluir que su utilización es adecuada en nuestro contexto. Es más, el hecho de haber usado la adaptación mencionada ha facilitado la comparación de los resultados, especialmente de la escala de estrategias de aprendizaje, con otros trabajos internacionales que empleaban la versión original ya que como se ha mencionado, la reproducen con mayor exactitud. Los valores de las alphas que se presentan a continuación corresponden a las obtenidas en este estudio. 
Está formado por 81 ítems divididos en dos secciones: una referente a la motivación y otra dedicada al aprendizaje. La sección de motivación consta de 31 ítems que evalúan la orientación a objetivos intrínsecos del alumnado (el grado en el que el/la estudiante percibe que participa en una tarea académica por razones como curiosidad, dominio de la materia, el hecho de que suponga un reto; 4 ítems, $\alpha=.71$ ); orientación a objetivos extrínsecos (el grado en el que el/la estudiante percibe que toma parte en una tarea por razones como las notas, posibles recompensas, la evaluación por parte de otras personas o la competencia; 4 ítems, $\alpha=.70$ ); valor concedido a la tarea (cómo de importante o útil es la tarea para el/la estudiante; 6 ítems, $\alpha=.84$ ); control de creencias de aprendizaje (el/la estudiante cree que sus esfuerzos por aprender le traerán buenos resultados; 4 ítems, $\alpha=.59$ ); autoeficacia para el aprendizaje y el desempeño (expectativas de éxito y autoeficacia; 8 ítems, $\alpha=.83$ ); ansiedad de test (preocupación y ansiedad por el desempeño; 5 ítems, $\alpha=.75$ ).

La sección de estrategias de aprendizaje consta de 50 ítems que evalúan el uso de estrategias de repetición (recitar o nombrar puntos en una tarea simple; 4 ítems, $\alpha=.67$ ); elaboración (estrategias como parafrasear, resumir, contrastar información, tomar notas; 6 ítems; $\alpha=.76$ ); organización (agrupación, subrayado y selección de la idea principal en fragmentos de lectura; 4 ítems, $\alpha=.74$ ); pensamiento crítico (aplicar conocimiento previo a nuevas situaciones o hacer evaluaciones críticas; 5 ítems, $\alpha=.77$ ); regulación metacognitiva (conciencia, conocimiento y control de la cognición; 12 ítems, $\alpha=.79$ ); tiempo de estudio y entorno (gestionar y regular el tiempo y el entorno/espacio; 8 ítems, $\alpha=.49$ ); regulación del esfuerzo (control del esfuerzo y atención frente a distracciones o una tarea poco interesante/aburrida; 4 ítems, $\alpha=.64$ ); aprendizaje entre iguales (colaboración entre iguales; 3 ítems, $\alpha=.63$ ) y búsqueda de ayuda (gestión de la ayuda de sus pares; 4 ítems, $\alpha=.40$ ). Las respuestas fueron obtenidas en una escala Likert de 7 puntos donde 1 correspondía a "totalmente en desacuerdo" y 7 "totalmente de acuerdo". Las puntuaciones de los ítems negativos fueron revertidas para que las puntuaciones más altas reflejasen una mayor orientación positiva y un uso más efectivo de las estrategias de aprendizaje. Las alphas obtenidas fueron similares a las de la versión original (Pintrich et al., 1991), aunque ligeramente inferiores en algunas de las subescalas.

Tabla 1

Escalas y subescalas del MSLQ (Pintrich et al., 1991)

\begin{tabular}{lll}
\hline Escala & Componente & \multicolumn{1}{c}{ Subescala } \\
\hline & & Orientación a objetivos intrínsecos \\
& Valor & Orientación a objetivos extrínsecos \\
& & Valor concedido a la tarea \\
Motivación & Control sobre creencias del conocimiento \\
& Expectativas & Autoeficacia para el aprendizaje y el \\
& & rendimiento \\
& Afectivo & Ansiedad ante los exámenes \\
\hline
\end{tabular}




\begin{tabular}{cll}
\hline Escala & \multicolumn{1}{c}{ Componente } & \multicolumn{1}{c}{ Subescala } \\
\hline & & Repetición \\
& Estrategias & Organización \\
& cognitivas y & Elaboración \\
Estrategias & metacognitivas & Pensamiento crítico \\
de aprendizaje & & Autorregulación metacognitiva \\
& & Gestión del tiempo y entorno de estudio \\
& Estrategias de & Regulación del esfuerzo \\
& control de recursos & Aprendizaje entre iguales \\
& & Búsqueda de ayuda \\
\hline
\end{tabular}

Nota: Elaboración propia

Desempeño académico. Se recabó información en torno al desempeño académico del alumnado a través de preguntas que recogían categorías implicadas en la autorregulación del aprendizaje: nivel de dificultad percibido (el grado de dificultad percibido por el/la estudiante, dividido en las siguientes categorías: "muy fácil", "fácil", "normal", "difícil", "muy difícil"), horas de dedicación semanal (número de horas dedicadas a tareas relacionadas con el Grado de Educación Primaria o Infantil en cada caso, dividido en "menos de dos horas", "entre dos y cinco horas", "entre seis y diez horas"y "más de diez horas"), asistencia (frecuencia con la que asisten a clase, conformado por las categorías "asistente habitual", "falto a veces", "falto a menudo") y trabajo (compaginación de los estudios con trabajar, reflejado en las categorías "unas horas a la semana", "media jornada" o, "jornada completa").

Rendimiento académico. El rendimiento académico se evaluó a través de las calificaciones del estudiantado. Dichas calificaciones se referían por un lado, a la nota de acceso a la universidad (dividida en las categorías de "de 5 a 7", "de 7 a 9", "de 9 a 10", "de 10 a 12" y "de 12 a 14). Esta calificación de acceso resulta clave debido a que todo el alumnado preuniversitario del territorio español debe pasar unas pruebas que influenciarán sus procesos de selección posteriores. Por el otro, a la nota media del grado (definida operativamente como media aritmética de las calificaciones obtenidas en todas las asignaturas de grado hasta el momento de la cumplimentación de la prueba, "menos de 5", “de 5 a 7", "de 7 a 9" y "de 9 a 10").

\section{Procedimiento de recogida y análisis de datos}

La cumplimentación de los cuestionarios se llevó a cabo de forma colectiva. Se explicó a los y a las estudiantes la naturaleza de la investigación y se les pidió su participación. La investigación atendió a los siguientes principios éticos: protección de los datos de carácter personal, protección de los derechos de voluntariedad, revocación, de confidencialidad, derecho a conocer los resultados, y derecho a decidir sobre el destino de los datos que figuran en el consentimiento informado.

El análisis de datos se llevó a cabo con el software estadístico IBM SPSS Statistics 24. En primer lugar, con el objetivo de analizar las diferencias en función del género 
y de la especialidad en las subescalas de aprendizaje autorregulado, se llevó a cabo un análisis multivariado de la varianza, siendo las variables dependientes las subescalas de aprendizaje autorregulado y las variables independientes la especialidad (Educación Infantil y Primaria) y el género. Para indagar en los efectos significativos aparecidos en función del género se procedió a una comparación de medias mediante la T-Student.

Posteriormente, con el propósito de analizar las diferencias en función del género y el rendimiento académico en el aprendizaje autorregulado, se llevaron a cabo dos análisis multivariados de la varianza, siendo las variables dependientes las subescalas de aprendizaje autorregulado y las variables independientes la nota de acceso y el género en el primer análisis y la nota de grado y el género en el segundo análisis. A continuación, mediante análisis univariados y pruebas post-hoc se indagó en las diferencias significativas específicas en función de la nota de acceso, la nota de grado y de la interacción entre género y nota de grado.

Los análisis se completaron explorando la asociación entre el género y variables de desempeño académico mediante el estadístico Ji cuadrado, que permite contrastar la relación entre variables categóricas.

\section{Resultados}

\section{Género, especialidad de magisterio y aprendizaje autorregulado}

Los resultados en la Traza de Pillai mostraron efectos estadísticamente significativos en función del género $(\mathrm{F}(3.423)=6.75 ; \mathrm{p}=.000)$ en muchas de las subescalas de aprendizaje autorregulado, pero no se hallaron efectos significativos ni en función de la especialidad $(\mathrm{F}(3.423)=1.650 ; \mathrm{p}=.059)$, ni de la interacción género*especialidad $(\mathrm{F}(3.423)=1.686$; $\mathrm{p}=0.51$ ). Estos resultados indican que las diferencias de género se mantienen en las dos especialidades de Educación.

Como se puede observar en la Tabla 1, con respecto a la dimensión de motivación, la prueba T-Student reveló que las estudiantes obtuvieron puntuaciones mayores que sus compañeros masculinos en las subescalas de motivación intrínseca, valor de la tarea, y ansiedad ante los exámenes. Los hombres puntuaron más alto en creencias de control.

En cuanto a la dimensión de estrategias de aprendizaje, los resultados de la prueba T-Student mostraron unas puntuaciones más elevadas a favor de las estudiantes en las subescalas de repaso, elaboración, organización, autorregulación metacognitiva, tiempo-espacio, regulación del esfuerzo, aprendizaje entre iguales y búsqueda de ayuda. 
Tabla 1

Diferencias de género en las dimensiones de motivación y estrategias de aprendizaje de aprendizaje autorregulado

\begin{tabular}{|c|c|c|c|c|c|c|}
\hline Escalas & $\begin{array}{l}\text { Chicas } \\
M(D T)\end{array}$ & $\begin{array}{l}\text { Chicos } \\
M(D T)\end{array}$ & G. L. & $T$ & $p$ & d-Cohen \\
\hline \multicolumn{7}{|l|}{ Motivación } \\
\hline Orientación a objetivos intrínsecos & $\begin{array}{c}21.1 \\
(3.44)\end{array}$ & $\begin{array}{l}20.18 \\
(3.92)\end{array}$ & 452 & -2.40 & .017 & 0.24 \\
\hline Orientación a objetivos extrínsecos & $\begin{array}{l}17.33 \\
(4.75)\end{array}$ & $\begin{array}{c}16.6 \\
(4.99)\end{array}$ & 452 & -1.41 & .157 & 0.14 \\
\hline Valor concedido a la tarea & $\begin{array}{l}31.41 \\
(5.33)\end{array}$ & $\begin{array}{l}29.23 \\
(6.59)\end{array}$ & 447 & -3.56 & .000 & 0.36 \\
\hline Creencias de control & $\begin{array}{l}19.65 \\
(3.58)\end{array}$ & $\begin{array}{l}20.58 \\
(3.81)\end{array}$ & 448 & 2.38 & .018 & 0.25 \\
\hline Creencias de autoeficacia & $\begin{array}{l}42.65 \\
(6.09)\end{array}$ & $\begin{array}{l}41.42 \\
(6.78)\end{array}$ & 450 & .055 & .067 & 0.19 \\
\hline Ansiedad de test & $\begin{array}{l}22.49 \\
(6.42)\end{array}$ & $\begin{array}{l}20.16 \\
(6.23)\end{array}$ & 450 & .349 & .001 & 0.36 \\
\hline \multicolumn{7}{|l|}{ Estrategias de aprendizaje } \\
\hline Repetición & $\begin{array}{l}20.96 \\
(4.27)\end{array}$ & $\begin{array}{c}18.5 \\
(4.98)\end{array}$ & 450 & 5.14 & .000 & .53 \\
\hline Elaboración & $\begin{array}{l}30.19 \\
(5.89)\end{array}$ & $\begin{array}{l}27.33 \\
(6.24)\end{array}$ & 448 & -4.45 & .000 & .47 \\
\hline Organización & $\begin{array}{l}22.04 \\
(4.11)\end{array}$ & $\begin{array}{l}17.80 \\
(5.13)\end{array}$ & 452 & -8.9 & .000 & .91 \\
\hline Pensamiento crítico & $\begin{array}{l}23.45 \\
(4.78)\end{array}$ & $\begin{array}{l}23.12 \\
(5.71)\end{array}$ & 452 & -.61 & .541 & .06 \\
\hline Autorregulación metacognitiva & $\begin{array}{l}58.38 \\
(8.65)\end{array}$ & $\begin{array}{c}51.96 \\
(12.75)\end{array}$ & 266 & -512 & .000 & .59 \\
\hline Tiempo y entorno & $\begin{array}{l}41.45 \\
(7.04)\end{array}$ & $\begin{array}{l}35.45 \\
(7.01)\end{array}$ & 448 & -6.55 & .000 & .85 \\
\hline Regulación del esfuerzo & $\begin{array}{l}20.22 \\
(4.19)\end{array}$ & $\begin{array}{l}17.58 \\
(4.71)\end{array}$ & 450 & -5.68 & .000 & .59 \\
\hline Aprendizaje entre iguales & $\begin{array}{l}13.81 \\
(3.63)\end{array}$ & $\begin{array}{l}11.88 \\
(3.52)\end{array}$ & 452 & -4.99 & .000 & .53 \\
\hline Búsqueda de ayuda & $\begin{array}{l}19.56 \\
(3.68)\end{array}$ & $\begin{array}{l}18.45 \\
(3.89)\end{array}$ & 448 & -2.75 & .006 & .29 \\
\hline
\end{tabular}

Nota: La diferencia de medias es significativa en el nivel .05 


\section{Nota de acceso y de grado, género, y aprendizaje autorregulado}

Los resultados en la Traza de Pillai revelaron efectos estadísticamente significativos en la nota de acceso $(\mathrm{F}(4.408)=1.79 ; \mathrm{p}=.000)$, en la nota de grado $(\mathrm{F}=(3.392)=1,75 ; \mathrm{p}=0.004)$, y en la interacción género*nota de grado $(\mathrm{F}(3.392)=1.43 ; \mathrm{p}=0.033)$ en las subescalas de aprendizaje autorregulado.

Diferencias en las notas de acceso. Los análisis univariados (pruebas post hoc) aportaron datos más específicos sobre las diferencias significativas entre los grupos en función de la nota. El alumnado con mejores puntuaciones de acceso obtuvo también puntuaciones más elevadas en varias subescalas de aprendizaje autorregulado. La Figura 1 ilustra que esta relación se manifiesta de manera progresiva, ya que a medida que aumenta la nota de acceso, de 5-7 hasta 12-14, también incrementa la puntuación en creencias de autoeficacia $\mathrm{F}(4.408)=2.82 ; \mathrm{p}=.025$ ) desde la $\mathrm{M}=38.75$ hasta la $\mathrm{M}=46.75$; en pensamiento crítico $\mathrm{F}(4.408)=3.69 ; \mathrm{p}=.006$ ) desde la $\mathrm{M}=20.87$ hasta la $\mathrm{M}=27.58$; en autorregulación metacognitiva $\mathrm{F}(4.408)=3.03 ; \mathrm{p}=.017$ desde la $\mathrm{M}=51.50$ hasta la $\mathrm{M}=56.42$ $y$ en aprendizaje entre iguales $\mathrm{F}(4.408)=3.52 ; \mathrm{p}=.008$ desde la $\mathrm{M}=8.75$ hasta la $\mathrm{M}=13.33$. Dada la abundancia de resultados significativos y con el fin de facilitar comprensión, estos se muestran en la figura a continuación:

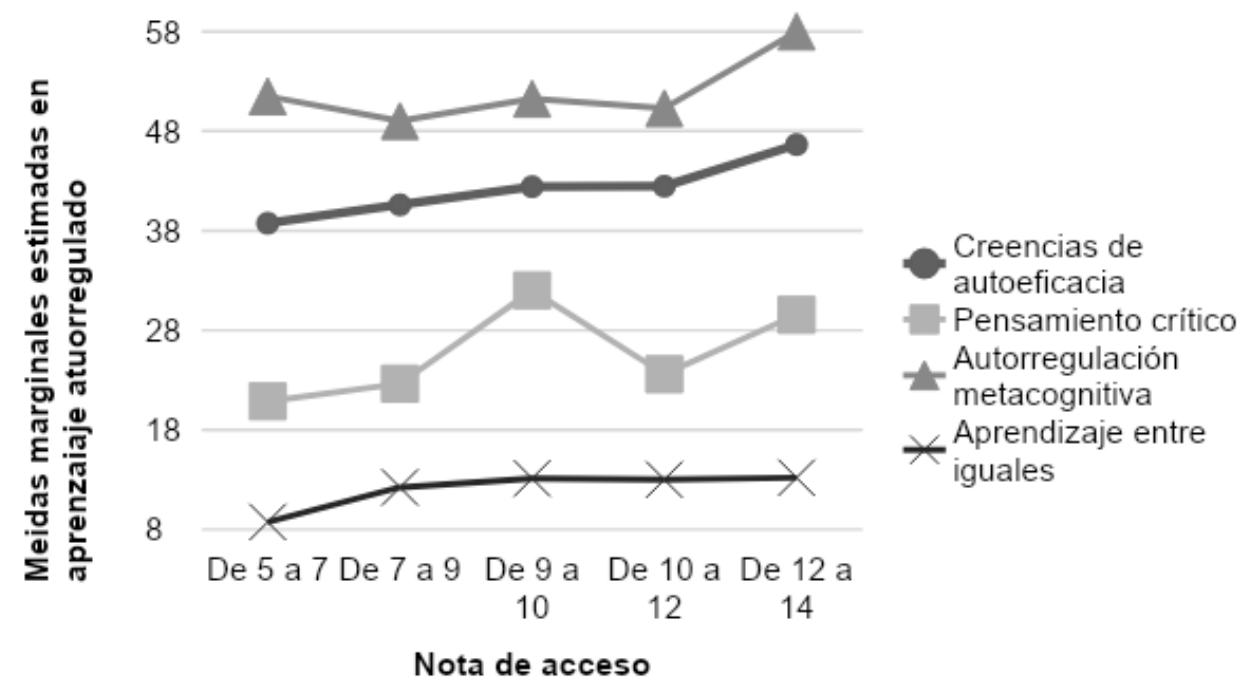

Figura 1. Diferencias en función de la nota de acceso

Diferencias en las notas de grado. Los análisis univariados mostraron la existencia de diferencias entre el alumnado según las notas de grado y las subescalas de motivación intrínseca $\mathrm{F}(3.384)=2.70 \mathrm{p}=.045)$, creencias de autoeficacia $\mathrm{F}(3.384)=5.73 ; \mathrm{p}=.001$ ); pensamiento crítico $\mathrm{F}(3.384)=2.70 ; \mathrm{p}=.045)$; regulación del esfuerzo $\mathrm{F}(3.384)=6.04 ; \mathrm{p}=.001$ y aprendizaje entre iguales $F(3.384)=4.95 ; p=.002$. Estos se presentan en la Figura 2. 


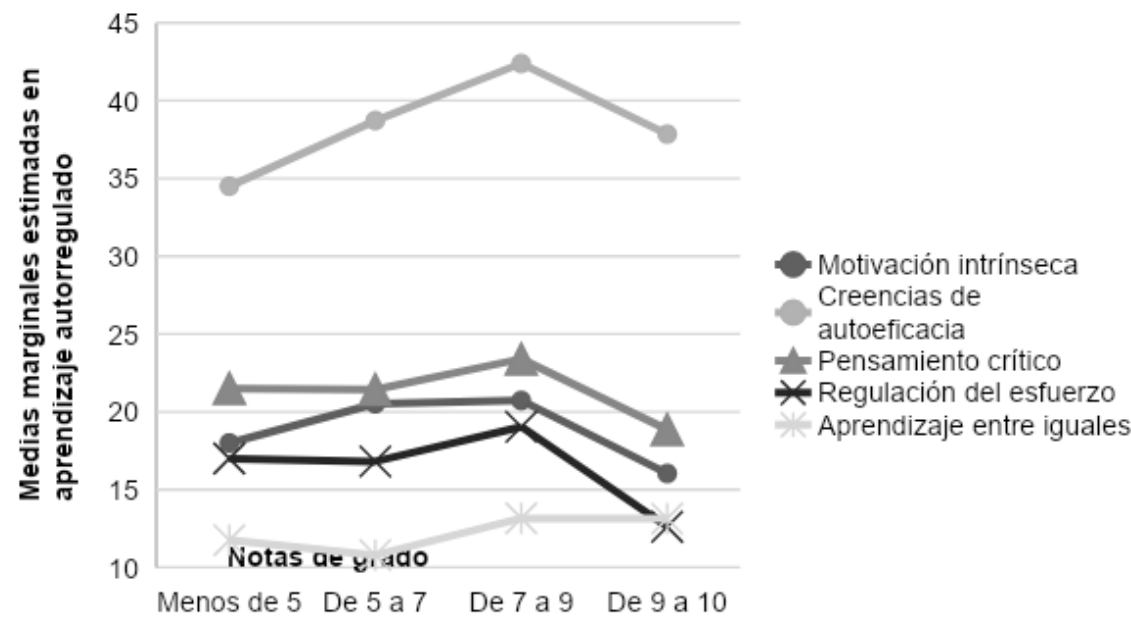

Figura 2. Diferencias en función de la nota de grado.

En comparación con las descritas en las notas de acceso, estas diferencias no siguen una línea claramente progresiva desde las puntuaciones del alumnado con peores notas (menos de 5) hasta las del alumnado con mejores puntuaciones en el grado (9-10). Se puede observar un declive del alumnado con mejores notas en las subescalas de motivación intrínseca, creencias de autoeficacia, pensamiento crítico y regulación del esfuerzo. Este declive no aparece en aprendizaje entre iguales.

Con el fin de conocer con exactitud los grupos en los que había diferencias significativas, se llevó a cabo la prueba de comparaciones múltiples entre pares de medias para los efectos simples de la interacción en las variables de motivación y estrategias de aprendizaje respectivamente, como se expone en las tablas 2 y 3 . Debido a su longitud, únicamente se han incluido las comparaciones que han resultado significativas.

Tabla 2

Prueba de Tukey de comparaciones post hoc por parejas de las subescalas de motivación en la nota de grado

\begin{tabular}{|c|c|c|c|c|c|c|c|}
\hline \multirow{2}{*}{ Escala } & \multirow{2}{*}{ Nota } & \multirow{2}{*}{\multicolumn{2}{|c|}{ Diferencia de medias }} & \multirow{2}{*}{$\begin{array}{c}\text { Error } \\
\text { estándar }\end{array}$} & \multirow{2}{*}{$p$} & \multicolumn{2}{|c|}{$\begin{array}{c}95 \% \text { de intervalo de } \\
\text { confianza para modelo }\end{array}$} \\
\hline & & & & & & $\begin{array}{l}\text { Límite } \\
\text { inferior }\end{array}$ & $\begin{array}{l}\text { Límite } \\
\text { superior }\end{array}$ \\
\hline \multicolumn{8}{|c|}{$\begin{array}{l}\text { Motivación } \\
\text { intrínseca }\end{array}$} \\
\hline & $5-7$ & $9-10$ & $4.482^{*}$ & 1.897 & .019 & .752 & 8.211 \\
\hline & $7-9$ & $9-10$ & 4.691 & 1.825 & .011 & 1.102 & 8.279 \\
\hline & \multirow{2}{*}{$9-10$} & $5-7$ & -4.482 & 1.897 & .019 & -8.211 & -.752 \\
\hline & & 7-9 & -4.691 & 1.825 & .011 & -8.279 & -1.102 \\
\hline
\end{tabular}




\begin{tabular}{|c|c|c|c|c|c|c|c|}
\hline \multirow{2}{*}{ Escala } & \multirow{2}{*}{ Nota } & \multirow{2}{*}{\multicolumn{2}{|c|}{ Diferencia de medias }} & \multirow{2}{*}{$\begin{array}{c}\text { Error } \\
\text { estándar }\end{array}$} & \multirow{2}{*}{$p$} & \multicolumn{2}{|c|}{$\begin{array}{l}95 \% \text { de intervalo de } \\
\text { confianza para modelo }\end{array}$} \\
\hline & & & & & & $\begin{array}{l}\text { Límite } \\
\text { inferior }\end{array}$ & $\begin{array}{c}\text { Límite } \\
\text { superior }\end{array}$ \\
\hline \multicolumn{8}{|c|}{$\begin{array}{l}\text { Creencias } \\
\text { autoeficacia }\end{array}$} \\
\hline & $<5$ & $7-9$ & -7.885 & 3.814 & .039 & -15.383 & -.386 \\
\hline & $5-7$ & $7-9$ & $-3.665^{*}$ & 1.062 & .001 & -5.753 & -1.577 \\
\hline & \multirow[t]{2}{*}{$7-9$} & $<5$ & $7.885^{*}$ & 3.814 & .039 & .386 & 15.383 \\
\hline & & $5-7$ & $3.665^{*}$ & 1.062 & .001 & 1.577 & 5.753 \\
\hline
\end{tabular}

Nota: ${ }^{*}$ Se basa en medidas marginales estimadas. ${ }^{* *}$ La diferencia de medias es significativa en el nivel .05.

Tabla 3

Prueba Tukey de comparaciones post hoc por parejas de las subescalas de estrategias de aprendizaje en la nota de grado

\begin{tabular}{|c|c|c|c|c|c|c|c|}
\hline \multirow{2}{*}{ Escala } & \multirow{2}{*}{ Nota } & \multirow{2}{*}{\multicolumn{2}{|c|}{ Diferencia de medias }} & \multirow{2}{*}{$\begin{array}{c}\text { Error } \\
\text { estándar }\end{array}$} & \multirow{2}{*}{ Sig. } & \multicolumn{2}{|c|}{$\begin{array}{c}95 \% \text { de intervalo de } \\
\text { confianza para modelo }\end{array}$} \\
\hline & & & & & & $\begin{array}{l}\text { Límite } \\
\text { inferior }\end{array}$ & $\begin{array}{c}\text { Límite } \\
\text { superior }\end{array}$ \\
\hline \multicolumn{8}{|c|}{ Pensamiento crítico } \\
\hline & 5-7 & $7-9$ & -1.961 & .863 & .024 & -3.659 & -.263 \\
\hline & $7-9$ & $5-7$ & 1.961 & .863 & .024 & .263 & 3.659 \\
\hline \multicolumn{8}{|c|}{ Regulación esfuerzo } \\
\hline & 5-7 & 7-9 & -2.229 & .718 & .002 & -3.641 & -.817 \\
\hline & & $5-7$ & 2.229 & .718 & .002 & .817 & 3.641 \\
\hline & $7-9$ & $9-10$ & 6.424 & 2.151 & .003 & 2.195 & 10.654 \\
\hline & 9-10 & $7-9$ & -6.424 & 2.151 & .003 & -10.654 & -2.195 \\
\hline \multicolumn{8}{|c|}{ Aprendizaje entre iguales } \\
\hline & 5-7 & 7-9 & -2.365 & .619 & .000 & -3.582 & -1.149 \\
\hline & $7-9$ & $5-7$ & 2.365 & .619 & .000 & 1.149 & 3.582 \\
\hline
\end{tabular}

Nota: * Se basa en medidas marginales estimadas. ${ }^{* *}$ La diferencia de medias es significativa en el nivel .05 .

Diferencias en la interacción entre género*nota de grado. Estas diferencias significativas se relacionaron con la motivación intrínseca, valor de la tarea, tiempo espacio y regulación del esfuerzo, a favor de las alumnas. Tal y como se puede observar en la figura 3, las mujeres presentan unas mejores habilidades autorregulatorias (a excepción del grupo ๑menos de 5๑) en diferentes componentes relacionados con la motivación y las estrategias de aprendizaje. 


\section{Desempeño académico y género}

A continuación se muestran los resultados significativos hallados en los diferentes componentes referentes al desempeño académico:

Tiempo de dedicación semanal. La prueba de Ji Cuadrado reveló que el género y el tiempo de dedicación semanal guardaban relación (Ji cuadrado (3) $=30.89$; $p=.000$; $\mathrm{V}_{\text {Cramer }}=.26$ ). El análisis de los residuos tipificados corregidos señaló que más chicos de los esperados invierten pocas horas de dedicación y más chicas de lo esperado invierten más horas de dedicación.
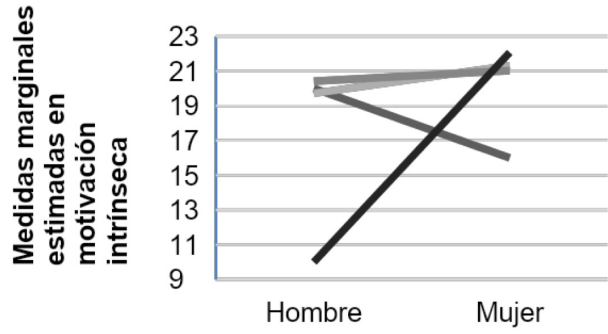

Género

Menos de 5

De 5 a 7

De 7 a 9

- 9 a 10

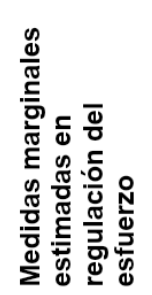

23
21
19
17
15
13
11
9
7
5
3

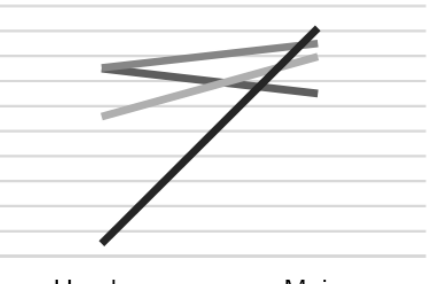

Hombre

Mujer

Género

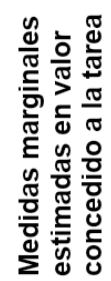

19.5

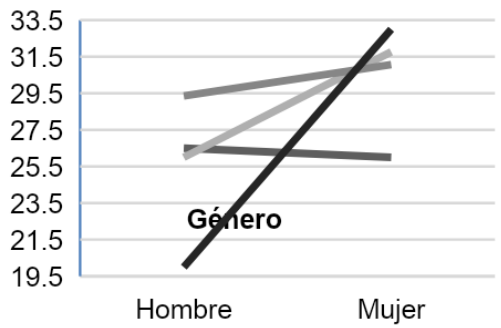

Menos de 5

- De 5 a 7

-

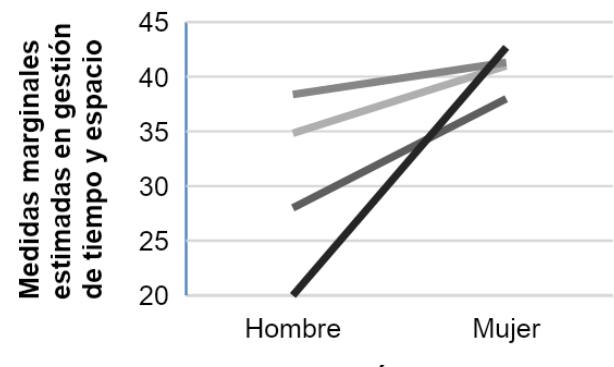

Género

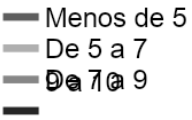

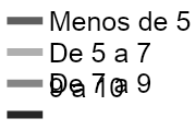

Figura 3. Efectos de la interacción género*nota de grado.

Dificultad percibida de la carrera. La prueba de Ji Cuadrado mostró que el género y la dificultad percibida también estaban relacionados (Ji cuadrado (5)=19.83; $\mathrm{p}=.001$; 
$\mathrm{V}_{\text {Cramer }}=.21$ ). En este caso, más alumnos de los esperados perciben la carrera con unos niveles de dificultad entre muy baja y normal, mientras que más alumnas de las esperadas la valoran como normal o incluso difícil.

Nota de acceso. La prueba de Ji Cuadrado manifestó que el género y la nota de acceso estaban relacionadas (Ji cuadrado $(4)=10.169 ; \mathrm{p}=.038 ; \mathrm{V}_{\text {Cramer }}=.15$ ). Los datos indican que más alumnos de los esperados obtienen notas bajas y menos de los esperados notas altas. Exactamente lo contrario que en el caso de las alumnas, ya que menor número de ellas obtienen notas bajas y mayor número notas altas.

Nota de grado. La prueba de Ji Cuadrado mostró que el género y la nota de grado están relacionadas (Ji cuadrado(3)=17.67; $\mathrm{p}=.001 ; \mathrm{V}_{\text {Cramer }}=.21$ ). Nuevamente, más alumnos de los esperados obtienen notas bajas y menos de los esperados notas altas. Al contrario que con las alumnas, ya que menos chicas de las esperadas obtienen notas bajas y más de las esperadas notas altas.

Finalmente, se constató que no existían relaciones significativas entre el género y la asistencia a clase (Ji cuadrado(2) $=5.64 ; \mathrm{p}=.059)$, ni entre el género y estar o no trabajando (Ji cuadrado(3)=6.87; $\mathrm{p}=.076$ ).

Tabla 4

Tabla de contingencia género*dedicación semanal, dificultad percibida, nota de acceso y nota de grado

\begin{tabular}{|c|c|c|c|c|c|c|c|}
\hline \multirow{3}{*}{$\begin{array}{l}\text { Desempeño } \\
\text { académico }\end{array}$} & \multicolumn{7}{|c|}{ Género } \\
\hline & \multicolumn{3}{|c|}{ Hombre } & \multicolumn{3}{|c|}{ Mujer } & \multirow[b]{2}{*}{$\mathrm{p}(\mathrm{z})$} \\
\hline & \multicolumn{2}{|c|}{ Recuento } & \multirow[t]{2}{*}{$\begin{array}{c}\text { Frecuencia } \\
\text { esperada }\end{array}$} & \multicolumn{2}{|c|}{ Recuento } & \multirow[t]{2}{*}{$\begin{array}{c}\text { Frecuencia } \\
\text { esperada }\end{array}$} & \\
\hline Dedicación & & & & & & & \\
\hline$<2 \mathrm{~h}$ & 36 & $30.5 \%$ & 18.8 & 36 & $10.7 \%$ & 53.2 & $<.05$ \\
\hline $2 h-5 h$ & 66 & $55.9 \%$ & 68.8 & 198 & $59.1 \%$ & 195.2 & $<.05$ \\
\hline $6 \mathrm{~h}-10 \mathrm{~h}$ & 14 & $11.9 \%$ & 26.6 & 88 & $26.3 \%$ & 75.4 & $<.05$ \\
\hline$>10 \mathrm{~h}$ & 2 & $1.7 \%$ & 3.9 & 13 & $3.9 \%$ & 11.1 & $>.05$ \\
\hline \multicolumn{8}{|l|}{ Dificultad } \\
\hline Muy fácil & 8 & $6.7 \%$ & 3.9 & 7 & $2.1 \%$ & 11.1 & $<.05$ \\
\hline Fácil & 37 & $31.1 \%$ & 24.1 & 55 & $16.4 \%$ & 67.9 & $<.05$ \\
\hline Normal & 70 & $58.8 \%$ & 85.7 & 257 & $76.7 \%$ & 241.3 & $<.05$ \\
\hline Difícil & 3 & $2.5 \%$ & 4.2 & 13 & $3.9 \%$ & 11.8 & $>.05$ \\
\hline Muy difícil & 1 & $0.8 \%$ & .8 & 2 & $0.6 \%$ & 2.2 & $>.05$ \\
\hline \multicolumn{8}{|l|}{ Nota acceso } \\
\hline $5-7$ & 4 & $3.4 \%$ & 2.4 & 5 & $1.5 \%$ & 6.6 & $>.05$ \\
\hline $7-9$ & 28 & $23.9 \%$ & 31.8 & 93 & $28.4 \%$ & 89.2 & $>.05$ \\
\hline $9-10$ & 61 & $52.1 \%$ & 48.9 & 125 & $38.1 \%$ & 137.1 & $<.05$ \\
\hline $10-12$ & 22 & $18.8 \%$ & 30.8 & 95 & $29 \%$ & 86.2 & $<.05$ \\
\hline $12-14$ & 2 & $1.7 \%$ & 3.2 & 10 & $3 \%$ & 8.8 & $>.05$ \\
\hline
\end{tabular}

Nota grado 


\begin{tabular}{|c|c|c|c|c|c|c|c|}
\hline \multirow{3}{*}{$\begin{array}{l}\text { Desempeño } \\
\text { académico }\end{array}$} & \multicolumn{7}{|c|}{ Género } \\
\hline & \multicolumn{3}{|c|}{ Hombre } & \multicolumn{3}{|c|}{ Mujer } & \multirow[b]{2}{*}{$\mathrm{p}(\mathrm{z})$} \\
\hline & \multicolumn{2}{|c|}{ Recuento } & \multirow{2}{*}{$\begin{array}{c}\begin{array}{c}\text { Frecuencia } \\
\text { esperada }\end{array} \\
.8\end{array}$} & \multicolumn{2}{|c|}{ Recuento } & \multirow{2}{*}{$\begin{array}{c}\begin{array}{c}\text { Frecuencia } \\
\text { esperada }\end{array} \\
2.2\end{array}$} & \\
\hline$<5$ & 2 & $1.9 \%$ & & 1 & $0.3 \%$ & & $>.05$ \\
\hline $5-7$ & 20 & $18.5 \%$ & 11.4 & 24 & $7.8 \%$ & 32.6 & $<.05$ \\
\hline $7-9$ & 84 & $77.8 \%$ & 87.8 & 254 & $82.5 \%$ & 250.3 & $>.05$ \\
\hline $9-10$ & 2 & $1.9 \%$ & 8 & 29 & $9.4 \%$ & 23 & $<.05$ \\
\hline
\end{tabular}

\section{Discusión y conclusiones}

\section{Discusión}

Las especificidades que a continuación se describirán atienden a la relación entre el género, el rendimiento académico, la especialidad de los grados de Educación y otras conductas del alumnado relativas a su desempeño académico.

\section{Género, especialidad y aprendizaje autorregulado}

Los resultados referentes al primer objetivo muestran una mayor autorregulación a favor de las estudiantes en gran parte de los componentes del aprendizaje autorregulado, siguiendo lo postulado en la primera hipótesis de este trabajo. No es la primera vez que se hallan estos resultados. Zimmerman (2010), referente clave en la teoría de la autorregulación en el aprendizaje, mostró resultados similares en algunos de sus estudios y los definió como provocativos. Sin embargo, a pesar de que los resultados de otras muchas investigaciones con respecto a las diferencias de género en los diferentes componentes de aprendizaje autorregulado apunten en la misma dirección, existen entre ellos diferencias en cuanto a la magnitud del tamaño del efecto de estas diferencias, con respecto a la inclusión o no de algunas de ellas y en relación a las posibles explicaciones sobre el origen de las mismas.

En este estudio, las diferencias de género significativas tienen un tamaño del efecto alto en las subescalas de organización y gestión del tiempo y entorno. Las diferencias en organización han sido halladas en otras investigaciones (Bidjerdano, 2005; Navea, 2015; Torrano \& Soria, 2017; Valenzuela \& Suárez, 2017), así como las referentes al contexto y gestión del estudio (Ablard \& Lipschultz, 1998; Bidjerdano, 2005; Suárez et al., 2004; Zimmerman \& Martínez-Pons, 1990).

Asimismo, se han encontrado diferencias de género de tamaño moderado en las siguientes estrategias de aprendizaje: repaso, autorregulación metacognitiva, regulación del esfuerzo y aprendizaje entre iguales. Éstas también coinciden con las halladas en varias investigaciones precedentes (Bidjerdano, 2005; Navea, 2015; Suárez et al., 2004).

Y finalmente, las diferencias de género de tamaño bajo corresponden a las subescalas de motivación intrínseca, valor concedido a la tarea, ansiedad ante los exámenes y elaboración, en línea con los resultados estudios previos (Albert, 2017; Bidjerdano, 2005; Navea, 2015; Rianudo et al., 2006; Rusillo \& Casanova, 2004; Yukselturk \& Bulut, 2009). 
Como se ha podido constatar, las diferencias mayores aparecen en estrategias de aprendizaje, aunque también existen en algunas subescalas de motivación con un tamaño del efecto más pequeño.

En lo que respecta a la especialidad, las diferencias se mantienen en los grupos de Educación Infantil y Educación Primaria, siendo más importante el género que la propia especialidad a la hora de explicar las diferencias en las habilidades autorregulatorias de estos grupos.

\section{Notas de acceso y de grado, género y aprendizaje autorregulado}

En consonancia con numerosas investigaciones previas, existe relación entre el rendimiento académico y las habilidades de autorregulación (Bozpolat, 2016; Salmerón et al., 2017; Torrano \& Soria, 2017), confirmándose así, lo planteado en la segunda hipótesis. En este estudio, se hizo una diferenciación entre las notas de acceso a la universidad y la nota de grado.

Desde el punto de vista general, tanto la nota de acceso a la universidad como la nota de grado están asociadas a muchas de las subescalas mencionadas y atienden a la siguiente relación gradual: cuanto mayor es la calificación, la puntuación en dichos componentes también es más elevada, en línea con lo mostrado en estudios previos (Bozopolat, 2016; Salmerón et al., 2017).

Gracias a la mencionada distinción entre las notas, se han hallado interesantes datos diferenciales. En lo que respecta a la nota de acceso, el alumnado con mejor calificación, también obtiene mayores puntuaciones en creencias de autoeficacia, autorregulación metacognitiva, pensamiento crítico y aprendizaje entre iguales, tanto en el caso de las mujeres como en el de los hombres.

Sin embargo, cuando dicho alumnado se encuentra ya en la universidad la nota de grado se asocia a motivación intrínseca, creencias de autoeficacia, pensamiento crítico, regulación del esfuerzo y aprendizaje entre iguales. Cabe destacar que el alumnado con mayor rendimiento académico disminuye sus puntuaciones en las mencionadas subescalas de aspectos más individuales de autorregulación cognitiva, para aumentar la puntuación en aprendizaje entre iguales.

Si tenemos en cuenta por un lado las reflexiones de Chocarro et al. (2007), sobre el impacto de las prácticas de enseñanza en la promoción de unas u otras competencias en el alumnado y por otro, el especial fomento del aprendizaje en grupo en el actual modelo de educación universitaria, estos datos del alumnado con mayor rendimiento no resultan extraños. Saben lo que hay que hacer en el nuevo contexto académico para obtener buen rendimiento y lo desarrollan. Sin embargo, lo que nos incita a una reflexión más profunda es que este aumento de aprendizaje entre iguales parece ir parejo a un detrimento de otras competencias más individuales de aprendizaje autorregulado que ya tenían adquiridas.

Los datos muestran que también existe una interacción entre el género y la nota de grado en cuatro de las subescalas relacionadas con la motivación intrínseca, el valor de la tarea, el tiempo-espacio y la regulación del esfuerzo. Estos datos conducen a la idea de que el grado motivase de manera más intrínseca a las estudiantes (Albert, 2017; Rusillo \& Casanova, 2004), valorasen más las tareas realizadas en el mismo, y poseyeran 
una mayor responsabilidad en la gestión del tiempo, espacio y esfuerzo. Los chicos, por su parte, muestran una motivación orientada a objetivos de carácter extrínseco.

Por lo tanto, todos los análisis desarrollados hasta el momento conducen a una imagen de las alumnas como más implicadas y comprometidas en los procesos de aprendizaje. Una posible explicación sería que las características autorregulatorias de las alumnas y los alumnos distan entre sí, lo que resulta de gran transcendencia a la hora de comprender los diferentes perfiles existentes en las aulas de los Grados de Educación Infantil y Primaria.

\section{Desempeño académico y género}

En consonancia con el apartado anterior y en lo referido a aspectos más globales del desempeño académico, también se ha hallado que este y el género están estrechamente vinculados en el caso del número de horas de trabajo dedicado a la semana, la dificultad percibida y las notas, tanto de acceso como de grado.

Los datos muestran que las alumnas perciben la carrera con mayor dificultad, dedican más horas fuera del aula y obtienen mejores notas de lo esperado. Por el contrario, los alumnos, perciben el grado como más fácil, dedican menos tiempo y su rendimiento académico es más bajo de lo esperado.

Como se puede observar, estos resultados nos muestran un panorama contradictorio que nos puede conducir a dos preguntas similares y complementarias entre sí: ¿cómo es posible que las alumnas consideren el grado con mayor dificultad e inviertan más horas a pesar de mostrar mejores habilidades autorregulatorias e incluso mejores notas? ¿Cómo es posible que los alumnos consideren el grado como más fácil a pesar de que se implican menos horas, muestran menores habilidades autorregulatorias e incluso peores notas?

Una posible explicación podría relacionarse con la manera de responder a los cuestionarios por parte de las chicas y los chicos, ya que existen evidencias de que los chicos sobreestiman sus capacidades mientras que las chicas son más realistas y modestas (Schunk \& Pajares, 2002; Torrano \& Soria, 2017). Sin embargo, no parece que este sea el único motivo que pueda explicar estos resultados porque, de hecho, hay diferencias significativas no solo en la percepción de dificultad sino también en las notas y el tiempo invertido fuera de clase.

Esta situación paradójica que se refleja en este y otros estudios recientes podría conducirnos a una reflexión que transciende el modo de responder a los cuestionarios y se ancla en los procesos de socialización diferenciales para chicos y chicas propios de nuestra sociedad.

\section{Conclusiones}

Los resultados de este estudio constituyen una notable contribución al análisis del género, las notas, la especialidad y el desempeño académico al analizar las diferencias individuales del alumnado.

En primer lugar, los hallazgos obtenidos sugieren que las mujeres poseen mejores habilidades de autorregulación tanto en las variables motivacionales como en las 
estrategias de aprendizaje, lo que las conduce a un mayor éxito académico. Dicha característica parece estar ligada al género, independientemente de la especialidad que se curse, lo que nos conduce a considerar la influencia de los procesos de aculturación social. Consecuentemente, cabe subrayar la importancia de seguir estudiando estas diferencias de género y los subyacentes procesos de socialización ya que como defienden Torrano et al. (2017), el análisis de estas se trata de una cuestión candente en la investigación socioeducativa debido a su impacto en la formación de las futuras generaciones.

Con respecto al rendimiento académico, se vuelve a confirmar su relación con los diferentes componentes de la teoría del aprendizaje autorregulado. Es más, se ha hallado que el alumnado con mejores notas de acceso a la universidad disminuye su regulación en varias de las subescalas, para aumentar en aprendizaje entre iguales. Esto podría deberse al nuevo modelo universitario, lo que nos conduce a la consideración de si este modelo ayuda a potenciar específicamente el aprendizaje entre iguales, pero en detrimento de otras habilidades.

En lo que al desempeño académico general respecta, se observa que las alumnas poseen un mayor rendimiento académico, una mayor dedicación y paradójicamente, también perciben el grado con mayor dificultad que sus compañeros. Estos resultados, como hemos mencionado previamente, requieren mayor investigación e incluso la inclusión de variables específicamente relacionadas con el peso de la socialización de género (valores interiorizados, estereotipos, interiorización de expectativas sociales e incluso niveles de consciencia de los procesos mentales) en la motivación, las estrategias de aprendizaje, las notas y el desempeño académico.

Asimismo, y en la línea del impacto de los factores contextuales (Ben-Eliyahu \& Bernacki, 2015) en el desarrollo de los procesos autorregulatorios, sería conveniente incluir en las investigaciones variables relacionadas con las características específicas del entorno universitario actual que son autopercibidas como muy relevantes por el propio alumnado, entre ellas, los trabajos de grupo, las exposiciones orales y los trabajos fin de grado (Larruzea-Urkixo, Cardeñoso e Idoiaga, 2019).

Todo lo anterior teniendo en mente que no habrá aprendices estratégicos sin maestros estratégicos (Chocarro et al., 2007; Peeters et al., 2014). Para ello es fundamental que desde las Facultades de Educación se promuevan los conocimientos y herramientas prácticas eficaces para la promoción de las habilidades autorregulatorias del alumnado universitario de Educación. De esta manera, ellos/as podrán ser modelos adecuados de profesor/a autorregulado en las aulas de Educación Infantil y Primaria.

Una de las limitaciones de este estudio podría ser que la muestra fue incidental y estuvo constituida por alumnado de primer y segundo curso. Una segunda limitación sería que la muestra se recogió en un único centro universitario. Otra limitación corresponde a la metodología empleada dado que el estudio fue abordado solamente con instrumentos cuantitativos. También sería interesante utilizar otros métodos de carácter cualitativo, como las técnicas thinking-aloud o las entrevistas, para así poder contrastar la información obtenida (Panadero, Jonsson \& Botella, 2017; Torrano \& Soria, 2017) y proporcionar una visión más global sobre la autorregulación académica del alumnado universitario. 
En resumen, partiendo de estas limitaciones y sugerencias de futuro, el análisis de las diferencias individuales en aprendizaje autorregulado podría ser enriquecido en futuros estudios incluyendo algunas de las variables contextuales mencionadas y con la participación de alumnado de otros cursos, grados y lugares, a fin de conocer si estos resultados también tienen lugar en otras condiciones y con ello mejorar la docencia en nuestras universidades

\section{Referencias}

Ablard, K., \& Lipschultz, R. (1998). Self-regulated learning in high achieving students: Relations to advanced reasoning, achievement goals and gender. Journal of Educational Psychology, 90(1), 94-101. https://doi:10.1037/0022-0663.90.1.94

Albert, A. (2017). Evaluación del aprendizaje autorregulado: Validación del motivated strategies for learning questionnaire en educación secundaria (Tesis Doctoral). Universitat de Vàlencia, Valencia.

Ben-Eliyahu, A., \& Bernacki, M.L. (2015). Addressing complexities in self-regulated learning: A focus on contextual factors, contingencies, and dynamic relations. Metacognition Learning, 10, 1-13. https://doi:10.1007/s11409-015-9134-6

Ben-Eliyahu, A., \& Linnenbrink-Garcia, L. (2015). Integrating the regulation of affect, behavior, and cognition into self-regulated learning paradigms among secondary and post-secondary students. Metacognition Learning, 10, 15-42. https://doi:10.1007/ s11409-014-9129-8

Bidjerano, T. (2005). Gender differences in self-regulated learning. Paper presented at the Annual Meeting of the Northeastern Educational Research Association, October 19-21, Kerhonkson, NY, USA.

Bozpolat, E. (2016). Investigation of the self-regulated learning strategies of students from the faculty of education using ordinal logistic regression analysis. Kuram Ve Uygulamada Egitim Bilimleri, 16(1), 301-318. https://doi:10.12738/estp.2016.1.0281

Broadbent, J., \& Poon, W. L. (2015). Self-regulated learning strategies \& academic achievement in online higher education learning environments: A systematic review. The Internet and Higher Education, 27, 1-13. http://dx.doi.org/10.1016/j.iheduc.2015.04.007

Bussey, K. (2011). The influence of gender on students' self-regulated learning and performance. In B. J. Zimmerman, \& D. H. Schunk (Eds.), Handbook of self-regulation of learning and performance (pp. 426-442). New York: Routledge.

Camoiras, Z., Benito, J. L, \& Varela, C. (2018). La motivación de los alumnos en la Educación Superior: evaluación de una experiencia docente. En A. Villa. (Ed.), Tendencias actuales de las transformaciones de las universidades en una nueva sociedad digital (pp. 631-374). Recuperado de http://www.foroinnovacionuniversitaria.net/ tendencias-actuales/

Cardozo, A. (2008). Motivación, aprendizaje y rendimiento académico en estudiantes del primer año universitario. Laurus, 14, 209-237.

Chocarro, E., González-Torres, M.C. \& Sobrino, J. (2007). Nuevas orientaciones en la formación del profesorado para una enseñanza centrada en la promoción del aprendizaje autorregulado de los alumnos. Estudios Sobre Educación, 12, 81-98. 
Dörrenbächer, L., \& Perels, F. (2016). Self-regulated learning profiles in college students: Their relationship to achievement, personality, and the effectiveness of an intervention to foster self-regulated learning. Learning and Individual Differences, 51, 229-241. https://doi.org/10.1016/j.lindif.2016.09.015.

García-Ros, R., \& Pérez-González, F. (2011). Validez predictiva e incremental de las habilidades de autorregulación sobre el éxito académico en la universidad. Revista De Psicodidáctica, 16, 231-250.

Karabenick, S.A., \& Zusho, A. (2015). Examining approaches to research on selfregulated learning: Conceptual and methodological considerations. Metacognition Learning, 10, 151-163. https://doi:10.1007/s11409-015-9137-3

Larruzea-Urkixo, N.; Cardeñoso Ramírez, O., e Idoiaga Mondragón, N. (2020). El alumnado del grado de educación ante las tareas universitarias: emoción y cognición. Educación XX1, 23(1), 197-220, https://doi: 10.5944/educXX1.23453

Muñoz, C. (2012). Relaciones existentes entre estrategias metacognitivas, motivación y rendimiento académico en los diferentes niveles educativos de estudiantes universitarios chilenos (Tesis Doctoral). Universidad del País Vasco, Leioa.

Navea, A. (2015). Un estudio sobre la motivación y estrategias de aprendizaje en estudiantes universitarios de ciencias de la salud (Tesis Doctoral). Universidad de Educación a Distancia, Madrid.

Panadero, E., Jonsson, A., \& Botella, J. (2017). Effects of self-assessment on self-regulated learning and self-efficacy: Four meta-analyses. Educational Research Review, 22, 74-98. https://doi.org/10.1016/j.edurev.2017.08.004

Peeters, J., Backer, F., Romero, V., Kindekens, A., Buffel, T., \& Lombaerts, K. (2014). The Role of Teachers' Self-regulatory Capacities in the Implementation of Self-regulated Learning Practices. Procedia - Social and Behavioral Sciences, 116, 1963-1970.

Perels, F., Dignath, C., \& Schmitz, B. (2009). Is it possible to improve mathematical achievement by means of self-regulation strategies? Evaluation of an intervention in regular math classes. European Journal of Psychology of Education, 24(1), 17-31.

Perry, N. E., \& Vandekamp, K. J. O. (2000). Creating classroom contexts that support young childrenos development of self-regulated learning. International Journal of Educational Research, 33, 821-843.

Pintrich, P. \& Smith, D. \& Duncan, T., \& Mckeachie, W. (1991). A Manual for the Use of the Motivated Strategies for Learning Questionnaire (MSLQ). Ann Arbor: Michigan.

Ponitz, C. C., McClelland, M. M., Matthews, J. S., \& Morrison, F. J. (2009). A structured observation of behavioral self-regulation and its contribution to kindergarten outcomes. Developmental Psychology, 45(3), 605-619. https://doi:10.1037/a0015365

Ramírez, M., \& Canto, J., \& Bueno, J., \& Echazarreta, A. (2013). Validación Psicométrica del Motivated Strategies for Learning Questionnaire en Universitarios Mexicanos. Electronic Journal of Research in Educational Psychology, 11(1), 193-214.

Randi, J., Corno, L., \& Johnson, E. (2011). Transitioning from college classroom to teaching career: Self-regulation in prospective teachers. New Directions for Teaching and Learning, 126, 89-98. https://doi:10.1002/tl.447

Rianudo, M. C., Barrera, M. L., \& Donolo, D. S. (2006). Motivación para el aprendizaje en alumnos universitarios. Revista Electrónica De Motivación y Emoción, 9(22), 1-19. 
Roces, C., Tourón, J., y González-Torres, M.C. (1995). “Validación preliminar del CEAM II (Cuestionario de Estrategias de Aprendizaje y Motivación II)". Psicológica, 16(3), 347-366.

Rusillo, M. T., \& Casanova, P. F. (2004). Diferencias de género en la motivación académica de los alumnos de educación secundaria obligatoria. Revista Electrónica De Investigación Psicoeducativa, 2(3), 97-112.

Salmerón, H., Gutiérrez-Braojos, C., \& Rodríguez, S. (2017). The relationship of gender, time orientation, and achieving self-regulated learning. Revista De Investigación Educativa, 35(2), 353-369.

Schunk, D. H., \& Pajares, F. (2002). The Development of Academic Self-Efficacy. In A. Wigfield, \& J. S. Eccles (Eds.), Development of Achievement Motivation (pp. 15-31). San Diego, CA: Academic Press. https://dx.doi.org/10.1016/B978-012750053-9/50003-6

Suárez, J. M., Anaya, D., \& Gómez, I. (2004). Diferencias diagnósticas en función del género respecto a la utilización de estrategias autorreguladoras en estudiantes universitarios. Revista De Investigación Educativa, 22(1), 245-258.

Torrano, F., Fuentes, J. L., \& Soria, M. (2017). Aprendizaje autorregulado: estado de la cuestión y retos psicopedagógicos. Perfiles educativos, 39(156), 160-173.

Torrano, F., y Soria, M. (2017). Diferencias de género y aprendizaje autorregulado: el efecto del rendimiento académico previo. Revista Complutense de Educación, 28(4), 1027-1042. https://dx.doi.org/10.5209/RCED.51096

Tseng, W. T., Liu, H., \& Nix, J. M. (2017). Self-regulation in language learning: Scale validation and gender effects. Perceptual and Motor Skills, 124(2), 531-548. https:// doi:10.1177/0031512516684293

Vargas, C. 2017. El aprendizaje a lo largo de toda la vida desde una perspectiva de justicia social. Serie de documentos temáticos sobre Investigación y Prospectiva en Educación, No. 21. París, UNESCO.

Yukselturk, E., \& Bulut, S. (2009). Gender differences in self-regulated online learning environment. Educational Technology \& Society, 12(3), 12-22.

Zimmerman, B. J. (2013). Theories of self-regulated learning and academic achievement: An overview and analysis. En B. J. Zimmerman, \& D. H. Schunk (Eds.), Self-regulated learning and academic achievement. Theoretical perspectives (2 $2^{\mathrm{a}}$ ed., pp. 1-37). Mahwah, N.J.: Lawrence Erbaum Associates, Inc.

Zimmerman, B. J., \& Martínez-Pons, M. (1990). Student differences in self-regulated learning: Relating grade, sex, and giftedness to self-eficacy and strategy use. Journal of Educational Psychology, 82(1), 51-59. https://doi:10.1037/0022-0663.82.1.51

Fecha de recepción: 16 de junio de 2018.

Fecha de revisión: 22 de junio de 2018.

Fecha de aceptación: 16 de diciembre de 2019. 
